\title{
Non-intrusive estimation of anisotropic stiffness maps of heterogeneous steel welds for the improvement of ultrasonic array inspection
}

\author{
Manuscript \\ Z. Fan ${ }^{1,3}$, A. F. Mark ${ }^{2,4}$, M. J. S. Lowe ${ }^{1}$, P. J. Withers ${ }^{2}$ \\ ${ }^{1}$ UK Research Centre in NDE \\ Department of Mechanical Engineering \\ Imperial College \\ London, SW7 2AZ, UK \\ ${ }^{2}$ School of Materials \\ University of Manchester \\ Grosvenor St, Manchester M13 9PL, UK \\ ${ }^{3}$ Now at school of Mechanical \& Aerospace Engineering \\ Nanyang Technological University \\ 50 Nanyang Avenue, Singapore 639798 \\ ${ }^{4}$ Now at Stuttgart Center for Electron Microscopy \\ Max Planck Institute for Intelligent Systems \\ Heisenbergstr. 3, 70569 Stuttgart, Germany
}




\begin{abstract}
It is challenging to inspect austenitic welds non-destructively using ultrasonic waves because the spatially varying elastic anisotropy of weld microstructures can lead to the deviation of ultrasound. Models have already been developed to predict the propagation of ultrasound in such welds once the weld stiffness heterogeneity is known. Consequently, it would be desirable to have a means of measuring the variation in elastic anisotropy experimentally so as to be able to correct for deviations in ultrasonic pathways for the improvement of weld inspection. This paper investigates the use of external non-intrusive ultrasonic array measurements to construct such weld stiffness maps, representing the orientation of the stiffness tensor according to location in the weld cross-section. An inverse model based on a genetic algorithm has been developed to recover a small number of key parameters in an approximate model of the weld map, making use of ultrasonic array measurements. The approximate model of the weld map uses the MINA formulation, which is one of the representations that has been proposed by other researchers to provide a simple, yet physically-based, description of the overall variations of orientations of the stiffness tensors over the weld cross-section. The choice of sensitive ultrasonic modes as well as the best monitoring positions have been discussed to achieve a robust inversion. Experiments have been carried out on a $60 \mathrm{~mm}$ thick multipass TIG (Tungsten Inert Gas) weld to validate the findings of the modelling, showing very good agreement. This work shows that ultrasonic array measurements can be used on a single side of a butt-welded plate, such that there is no need to access the remote side, to construct an approximate but useful weld map of the spatial variations in anisotropic stiffness orientation that occur within the weld.
\end{abstract}




\section{Introduction}

Austenitic steels are popular in the petro-chemical and nuclear industries, due to their excellent corrosion resistance, relatively high ductility and high strength compared to typical carbon steels. However, it is challenging to inspect welded joints in austenitic steel components using ultrasonic waves because of the problem of beam deviation arising from the spatial variation in elastic anisotropy local to the weld. This is caused by the polycrystal grain texture that develops within the weld metal during solidification from the molten state. The deviation results in errors in the interpretation of signals, poor array images, and incorrect defect sizing. Mathematical modeling based both on ray-tracing algorithms $[1,2,3,4]$ and finite element models [5, 6] has been carried out to study the propagation of ultrasound through welds having high degrees of inhomogeneity. More recently array imaging in such anisotropic inhomogeneous materials has also been performed providing an opportunity to correct the images in these difficult materials [3].

Critical to the analysis of welds by ultrasound is a weld anisotropy or stiffness map, which we will refer to in this article simply as a "weld map". This describes the spatial variations of the anisotropic elastic properties over the cross section of the weld and can be used to correct the beam paths. Such a map can be either simulated $[1,2,3]$ or measured experimentally $[5,6]$. Experimental measurements are usually based on microstructural analysis of post-mortem macrographs of weld cross-sections. However, in practice the weld map is a function of the weld process conditions so that it varies from case to case, and it is not practical to take destructive measurements of each weld sample. Therefore it is useful to develop a non-destructive method to characterize the weld, that can be used at the time of manufacture or during in-service inspections. In order to achieve a robust inversion, we propose that it is best for the weld to be described by a model with a modest number 
of key parameters. A number of models have been developed to describe the weld map using a small number of parameters, and these are reviewed in Section 2 of this paper. Among these models, a model named Modeling of anIsotropy based on Notebook of Arc-welding (MINA [6]) is based on information about the welding procedure and rules for polycrystal growth, and therefore provides a more physical approach to describe the real grain structure than the empirical geometry-based maps.

An important point must be made here concerning the nature of these weld maps. All of the cited weld maps in the literature aim to use a small number of parameters, such that the spatial variations of stiffness properties are represented in a general sense. This approach is an approximation because real welds include significant complexity in the patterns of stiffness properties at small scale, which for accurate maps would require a large number of parameters. However, the objective of using such a map to enable the correction of beam deviation in array imaging depends much more on the general variations than on the local ones. Furthermore, the inversion of measurements to find a small number of parameters is inherently more robust and computationally cheaper than for a large number of parameters. Therefore, while we must recognise that any model with a small number of parameters can only be an approximation, we propose that this is a sensible approach for a practical improvement of the array imaging inspection of inhomogeneous welds. It should also be kept in mind that the various models that are proposed for weld maps are themselves approximations, based usually on simple geometry, but sometimes on physical phenomena (as in the case of MINA), so they are not strictly correct even in the general sense, but they should certainly offer major improvements over preceding simple assumptions of homogeneity and isotropy, as has been shown in the validations in the publications relating to these models. 
A number of studies have already been carried out to investigate the construction of the weld map. Gueudre et al.[7] demonstrated a modeling procedure able to identify the parameters in the MINA model, from comparing the echo-dynamic curve of the signals received along a length on the base face of the weld; this is the amplitude profile across the receiver locations. This approach was validated by a simulated experiment. This is an interesting approach, however in practise it can be difficult to measure the echo dynamic curve accurately because the amplitude of the signal is affected particularly by the attenuation caused by the scattering in the weld material and also by the coupling of the receiving elements, both of which are uncertain. Indeed the authors chose to use a simulated experiment so that they could demonstrate the possibility while avoiding, for the present, these experimental challenges. More recently, Zhang et al. [8] presented another inversion approach. They divided the weld geometry into a finite number of mesh grids, and measured the time of flight result of longitudinal waves propagating through the weld using a shortest-path algorithm [9]. Subsequently a Monte Carlo Markov Chain method was used to refine the orientation of the grain in each element in the grid. This inversion method is more robust as it takes account of the time of flight of the ultrasound instead of the amplitude, however the inversion process is very slow as there are hundreds of unknown variables in the model. A new method based only upon a small number of key parameters to describe the weld anisotropy would benefit from a reduced computation time and ensure its industrial viability for use on real samples.

In previous models the ultrasonic signals have typically been generated on top of the weld, and received on the back face, allowing the ultrasound to cover most regions in the weld with ease. However, in practice access to the back face of the weld is usually limited and furthermore weld caps which are normally not removed after the welding process can interfere with 
the measurement process. Therefore an alternative approach is considered in our work here, in which two ultrasonic arrays are placed on the top surface of the sample, one on each side of the weld. The ultrasound is generated from one array, passes through the weld, is reflected from the back face and then received by the other array. Figure 1 shows a schematic of the arrangement, illustrating an example ray path from one element on the transmitter array to one on the receiver array. The multiple ray paths between pairs of array elements cover different directions and regions of the weld cross section, so enabling an inversion to extract the weld map. This is done using an optimization technique.

It is also noted that in previous studies, only the longitudinal mode has been used to find the weld map, which might not be the mode most sensitive to the material properties. In our study, detailed discussions on the best ultrasonic modes as well as array positions will be presented, which will contribute to a more robust inversion method.

This paper starts with a review of the models describing weld maps that are available in the literature, and discusses the choice of the most representative model based on the welding process. Section 3 presents a raytracing method that has been developed to model ultrasonic wave propagation through the inhomogeneous and anisotropic weld. In section 4, Finite Element (FE) simulations are presented to identify the most sensitive ultrasonic mode and best array positions. An inversion method, based on a global optimization algorithm using selected ultrasound signals from FE simulations is also presented in this section. Finally experimental results from a real weld sample are presented in section 5 . 


\section{Modeling of weld maps}

Real welds in thick-section plates or pipes are normally made using a large numbers of passes, and their solidification results in very complex spatial variations of elastic properties arising from the anisotropic nature of the single crystal stiffness tensor of the austenitic steel. Figure 2(a) shows a macrograph of a cross-section through a weld sample provided by our industrial partners. The sample contains a V-weld joining two 304L austenitic stainless steel plates of $60 \mathrm{~mm}$ thickness. A 308 stainless steel consumable root insert and filler wire were used to form the weld. Manual TIG welding was used to form the root of the weld, followed by multiple passes of automated orbital TIG until full-fill. The plates were horizontal when the weld was made. The macrograph was obtained using a Nikon D1X digital SLR camera with a Nikkor $105 \mathrm{~mm}$ macro lens, after polishing and etching the specimen. Within image processing software (Adobe Illustrator) the array of lines was overlaid on the macrograph; each arrow was then manually aligned with the grain structure beneath it. This was intended to be a simple, rapid method of obtaining an initial orientation map. The grey/white streaks in the macrograph indicate common directions of the crystal axes.

A common way (adopted by all of the cases that will be discussed here) of simplifying the description of the anisotropic material in the weld is to consider it to be transversely isotropic, in which case the plane perpendicular to the direction of the grain growth is considered to be isotropic, with the direction of the grain growth assumed to lie within the plane of the welded cross-section. This is known to be strictly incorrect, because the welding wire moves along the weld line (normal to this plane), so the heat flow and solidification direction are tilted out of this plane. There is also an influence of the orientation of the component while the weld is being made, when gravitational forces can skew the alignment of the solidifying material; this 
has a particularly large effect when the weld is vertical. Nevertheless this simplifying assumption of material symmetry has been shown to be approximately correct from macrograph and Electron Back Scattering Diffraction (EBSD) measurements [10], and has been adopted widely [5, 6, 3, 7, 8, 11]. The elastic constants are assumed to be the same everywhere within the weld, with the only variable parameter being the orientation of the unique material property axis in the plane of the cross section shown in Figure 2(b).

A number of models each involving only a few parameters to characterise the stiffness map have been developed. They have been discussed and compared by Apfel et al. [11], and so will only be briefly reviewed here. Ogilvy $[1,12]$ used empirical analytical functions to describe the continuous variation in anisotropy across a weld, and this model has been used by other researchers to model the weld [3]. Langerberg et al. [13] further simplified the structure and assumed the grains to be oriented at an angle of $45^{\circ}$ to the vertical axis. Spies et al. [14] divided the inhomogeneous region into layers of transversely isotropic material, with the orientation in each layer considered to be the same. Another common approach is to divide the weld geometry into a number of homogeneous sections, each having fixed orientation of the grains $[15,16,4]$. Most of these models only take into account the boundary geometry of the fusion zone without considering other local geometry such as the grain structure associated with each pass, thus they cannot always capture the complexity of a heterogeneous structure resulting from multi-pass arc welding. FE models can provide a more precise definition of these details inside the weld, but at cost, as FE models are relatively time consuming to build and solve. Our interest here is to develop a weld model having a small number of key parameters, that is not intended to be strictly precise in local detail, but that is sensibly representative of actual weld material and quick to run. 
A model named Modeling of anIsotropy based on Notebook of Arcwelding (MINA) has been developed by researchers in France to predict the weld stiffness map for shielded metal arc welding from physical information about the formation of the weld that would typically be documented by the welder [6]; it is now in use in relation to ultrasound inspection of power plant components [17]. It thus has a good physical foundation and validation in its context. A schematic of the MINA model is shown in Figure 3. The information it takes from the welding procedure includes the dimensions of the weld pool, dimension of the electrodes, the number of layers, and the number and order of passes in each layer. It also considers parameters that affect the direction of grain growth such as the inclination angle of the weld pass towards the weld groove $\theta_{B}$ or another weld pass $\theta_{C}$, and two remelting rates which describe the overlapping of weld passes in the vertical $\left(R_{v}\right)$ and lateral $\left(R_{l}\right)$ directions. The physical phenomena describing the solidification mechanism, which include the influence of temperature gradients in a weld pass and the epitaxy and competition between grains, are then considered iteratively in the modeling to obtain the global orientation map of the weld.

The example weld that we use for the modelling and experimental work in this study was constructed by manual and automated TIG, which leaves it strictly outside the specific context of shielded metal arc welding for which the MINA model was developed. However, it remains attractive because it is based on physical phenomena rather than geometric fitting to macrographs, and so has been selected for the inversion task in this work. Of course the proof comes from the comparison of the results, and we have found that this model, with appropriate parameters, is capable of delivering a good representation of both the macrograph geometry and ultrasonic performance. This is perhaps to be expected given the common thermal processes driving the formation of many kinds of welds and, critically, the fact that we are 
not pursuing accuracy at fine scale. Thus, using an implementation of the MINA model reported in [6], we have identified the following four MINA parameters $\theta_{B}=17.5^{\circ}, \theta_{C}=0, R_{v}=0.15, R_{l}=0.335$ for the weld of Figure 2. This was done by an optimization process comparing the weldmap from the MINA model and the grain orientations inferred from the macrograph that are shown in part (b) of the figure. We note that other parameters of the MINA model may also have significant influence on the weld map, for example Gueudre et al. [18] identify the order of the weld passes to be important; however, in the interests of simplicity over accuracy of detail, we have limited our study to these four parameters. Future work could extend our inversion process to additional parameters without difficulty if thought to be useful.

\section{Ray-tracing technique}

Our inversion procedure will use simulations of ray paths in the weld, iterating the parameters of the MINA model of the weld to achieve a target weld map. The multiple calculations of ray paths at the core of the inversion algorithm will require a fast technique, for which we have selected ray tracing. Ray-tracing techniques have been widely used in the modelling of seismic waves [19] and ultrasound [1, 2, 3, 4] to calculate wave propagation through an inhomogeneous medium. We have adopted the ray-tracing procedure developed in [3], which is summarised briefly here.

The ultrasonic rays passing through the weld follow a curved path as dictated by the varying orientations of the elastic constants of the material. In our model, a ray originates on the top surface on one side of the weld with a given phase vector, and a time-stepping calculation is used to take it through the weld. It is assumed that in each time step, the ultrasound propagates in a locally homogeneous and anisotropic medium, which is governed by the 
Christoffel equation:

$$
C_{i j k l} k_{j} k_{k}=\rho \omega^{2} \delta_{i l}
$$

where $C_{i j k l}$ is the stiffness tensor matrix of the material, $k$ is the wave vector, $\rho$ is the density and $\omega$ is the frequency. $\delta_{i l}$ is the Dirac delta function. The number of homogeneous equations, roots and velocities is equal to the number of spatial dimensions in the system. Given that the phase velocity $c_{p}=\omega / k$, equation 1 can be simplified as:

$$
\left|\rho c_{p}^{2} \delta_{i l}-\Gamma_{i l}\right|=0
$$

where $\Gamma=C_{i j k l} n_{j} n_{k}$ is called the Green-Christoffel acoustic tensor with $n$ being a vector describing the direction cosines of the wavevector. This is an eigensystem and the associate eigenvalues $\lambda$ and eigenvectors $v$ for a given slowness vector $m=k / \omega$ are:

$$
\lambda_{a}=\rho / m_{a}^{2} ; v_{a}=p_{a}
$$

where $1 \leq a \leq 3$, and $p$ is the polarisation vector. The group velocity $c_{g}$ can be derived as:

$$
c_{g a}=\frac{1}{\rho} C_{a j k l} p_{j} p_{k} m_{l}
$$

After each time step of the ray calculation, a non-physical boundary is applied locally in the model to account for the variation in the material properties. The orientation of the boundary is assumed to lie parallel to a line joining points of constant elastic constant orientation and passing through the ray's current position. The reflected and refracted waves at the local boundary can be computed by the sextic equation [20], and the direction of 
the ray is determined by the wave component with maximum energy; this is necessary because the calculation at the boundary results, in general, in multiple components of reflected and transmitted waves. This process is repeated until the ray leaves the inhomogeneous region.

Based on this ray-tracing model, simulations can be made to predict the paths and travel times for rays leaving any chosen transducer element at any chosen initial ("shooting") angle. An example of this, for SV (polarized in the plane of the cross-section) shear waves leaving a single source location, repeated multiple times to cover a range of angles, is shown in Figure 4. The material properties of the weld and the parent steel plates are listed in Table 1, with the former taken from the literature [21]. The weld map defining the orientation of these material properties at each location along the ray path was given by the MINA model with the parameters that were identified for the example weld and stated in the preceding section. The source location is on the top surface of the weld $40 \mathrm{~mm}$ to the right of the center of the weld, the initial (shooting) angles vary from $20^{\circ}$ to $70^{\circ}$ to the perpendicular, and the rays terminate at the backwall of the weld. Clear beam deviation of the rays when they are inside the weld can be observed from Figure 4(a). For comparison, rays generated at the same position going through isotropic steel are plotted in Figure 4(b). Figure 4(c) compares the times of flight of all the rays to reach their termination positions. Significant differences can be seen when comparing the times of flight for the rays passing through the weld with those at the same termination positions but for the isotropic steel. This is due to the deviation of the rays as well as the changes of wave speed along the ray paths. It is these differences that provide the basis for the inversion. The ray model is analytical and quick to compute, so it provides an ideal tool for multiple-repeated simulations at the core of the inversion process. 


\section{Inversion studies using Finite Element sim- ulations}

\subsection{FE model description}

Finite Element (FE) wave propagation modelling has been used in this work for three purposes: to validate the accuracy of the ray tracing models; to provide ideal noise-free "measurement" data sets to aid the development of the inversion process and select its setup and parameters; and to validate the inversion.

Figure 5 shows a schematic of the FE model, which has been run using the commercial software package ABAQUS/Explict [22]. The profile of the sample was measured from the real weld discussed earlier, which was provided by the industrial partners, and the orientation of the stiffness matrix in each element inside the weld follows the weld map defined by the same four MINA parameters $\theta_{B}=17.5^{\circ}, \theta_{C}=0, R_{v}=0.15, R_{l}=0.335$. The mesh size in the MINA model was chosen to be $0.5 \times 0.5 \mathrm{~mm}^{2}$. For simplification, the geometry and properties of the structure were mirrored with respect to the back face (the lower surface of the weld in the figure) of the weld so that mode conversions and edge waves at the back face were excluded. Automatically generated linear triangular elements (CPE3R) having a maximum length of $0.2 \mathrm{~mm}$ were applied in the model. Different material orientations were assigned to each element according to the weld map. Absorbing layers of $20 \mathrm{~mm}$ width were applied on the outside border of the model to suppress reflections from the boundary [23]. The model was excited by applying a time-varying force in the vertical direction on the top surface on the right hand side of the weld, which generated both shear and longitudinal wave modes; the shear modes were polarised in the plane of the cross-section (SV modes). The input force signal was a 3-cycle tone burst signal centered at 
$1 \mathrm{MHz}$. A number of monitoring locations were defined along the receiving surface, ranging in location from $-140 \mathrm{~mm}$ to $80 \mathrm{~mm}$ as shown in Figure 5.

\subsection{Selection of ultrasound mode and receiver posi- tions}

To assess the effect of the inhomogeneous weld material, a comparison FE model was also run using isotropic steel material throughout. Two example monitor locations are selected here to illustrate and explain the observations, and the monitored signals for these locations are shown in Figure 6. In both cases, a point source was placed on the top surface $30 \mathrm{~mm}$ to the right of the center of the weld. Figure 6(a) and (b) show received signals distant from the weld center at $-100 \mathrm{~mm}$, based on isotropic material properties and on those for weld material respectively. Figure 6(c) and (d) are the corresponding signals at a monitoring location very close to the weld cap, $-22 \mathrm{~mm}$. The signals for these two locations have both passed through the weld material, but on different paths. Both the longitudinal mode and the shear mode were generated and picked up at the monitoring locations and they can be distinguished by their arrival times.

The sensitivity of the ultrasonic modes to the weld material can be assessed by comparing the change in time of flight between waves that propagated through the weld and through the parent material. For example, by comparing Figure 6(a) with (b), it can be seen that the time shift of the longitudinal mode is less than $0.2 \mu \mathrm{s}$, while for the shear mode it is around 1.5 $\mu s$; this was a typical finding for any of the source or monitoring positions.

It is also notable in both examples that there are mode conversions between the longitudinal and the shear mode inside the weld, and the converted signal appears in between the two dominant modes in Figures 6(b) and (d). Figure $6(\mathrm{c})$ and $(\mathrm{d})$ shows the received signal at a monitor location that 
is placed very close to the weld cap. The mode conversion in Figure 6(d), when the monitoring location is close to the weld cap, is particularly strong, and furthermore the shear mode arrives at a similar time as the converted mode which makes it difficult to be separated. This suggests that the monitored region has to be selected carefully to obtain dependable signals for the inversion.

Figure 7 shows time of flight results for both shear and longitudinal modes, and for the weld material model and isotropic material model. The generator is at $30 \mathrm{~mm}$ as before, and there are a number of monitoring positions from $-140 \mathrm{~mm}$ to $80 \mathrm{~mm}$. Ray-tracing results with ultrasound wave propagation through the weld are also shown below the figure for illustration. Three zones have been defined for better interpretation of the results. In zone 1, the monitors were placed to the right of the generator, in which case the ultrasound propagates through isotropic material only in both models, and the time of flight results are found to be exactly the same, as expected. In zone 2 , the monitors were placed on the left side of the weld and some distance away from the center. A clear shift of the arrival time is observed in the shear mode in Figure 7(a) and the time difference obtained in this zone can be around 1-2 $\mu s$, which indicates good sensitivity to the weld map. In contrast, Figure $7(\mathrm{~b})$ shows that the time difference of the longitudinal mode in the same zone is very small, which possibly falls into the region of experimental error, therefore this mode would be more difficult to deploy for the inversion process. In zone 3, monitors were placed close to the center of the weld. In this region, there are large variations in time of flight results from signals propagating through the weld, especially with the shear mode. The reason can be understood from the signals in Figure 6(d). Due to strong mode conversions, the shear mode is very weak and difficult to be separated from the mode converted signals, therefore it is not reliable to use the shear 
mode in zone 3 for weld characterizations.

Thus it has been demonstrated that the shear mode in zone 2 is most sensitive to the inhomogeneity imposed by the weld map, and a zoom of this part of Figure 7(a) is shown by the solid line in Figure 8. For comparison, time of flight results calculated from ray-tracing with the same MINA parameters as in the FE simulations are shown in the same figure by the dashed line. This line was obtained by performing an angular sweep from the same generation position as in the FE model and recording the time of flight results of each of the rays when they terminate at the surface of the weld sample. Very good agreement has been observed between the results obtained from ray-tracing models and the FE simulations for the same weld map. For comparison, time of flight results from another two weld maps, generated using alternative, but reasonable, MINA parameters, are presented in the same figure, showing a significant difference. This confirms again that the shear mode is sensitive to the material properties inside the weld.

The outcome of these studies was the selection of the shear mode, with monitoring in zone 2, to be used for the inversion process to find the weld map.

\subsection{Inversion technique based on a Genetic Algorithm}

Since the weld stiffness map has been described by a small number of key parameters, and these have been shown to have significant influence on the travel time on well-chosen ray paths and the shear wave mode, we have established a good basis to attempt a well conditioned inversion process to determine these parameters from ultrasonic measurements. This section describes the inversion model used to refine the parameters in the MINA model from the ultrasonic array measurements. It is based on a global optimization process, and an iterative algorithm has been applied to compare the results 
from the forward model with the experiment. The inversion process is illustrated in Figure 9. This is similar to the approach that was applied in the work by Gueudre et al. [7], which iteratively compares the echo dynamic curve obtained from a Finite Element simulation [24] and a simulated experiment. However there is a significant difference in that our approach is based on the arrival time at chosen locations whereas the approach by Gueudre et al. uses the profile of the signal amplitude across the receiver locations. We expect our approach to be less sensitive to the uncertainties of attenuation in the weld material and transducer coupling.

In the inverse model, a fitness function has been used to quantify the similarity between experiments and simulations, which can be expressed in the sense of least squares:

$$
J\left(\theta_{B}, \theta_{C}, R_{v}, R_{l}\right)=\sum_{i=1}^{n}\left(y_{i}^{s i m}\left(\theta_{B}, \theta_{C}, R_{v}, R_{l}\right)-y_{i}^{e x p}\right)^{2}
$$

where $\theta_{B}, \theta_{C}, R_{v}, R_{l}$ are the four parameters applied in the MINA model, $y_{i}^{\text {sim }}$ and $y_{i}^{e x p}$ represent the time of flight results by simulations and experiments respectively and $n$ corresponds to the number of rays. A genetic algorithm [25], which is based on the Darwin natural evolution theory, was implemented to find the global minimum in the fitness function. The algorithm starts with an initialized population of individuals, each of which corresponds to a set of four parameters $\theta_{B}, \theta_{C}, R_{v}, R_{l}$, and is characterized by the fitness function. Based on their fitness, parents are selected to produce a new generation using operators such as crossover, mutation and replacements. If the system is well designed and well conditioned, the population will tend to converge to a global optimal solution. The algorithm has been developed in Fortran, by integrating the ray-tracing model with an open source code of the genetic algorithm [26]. In our model, the population of the model has been chosen to 
be 20 sets of four MINA parameters, the crossover rate was chosen to be 0.6 and the mutation rate was 0.02 to achieve the best efficiency of the algorithm. The following boundaries, estimated from the analysis of the macrograph, were applied to the four MINA parameters: $\theta_{B}$ and $\theta_{C}$ in between $0^{\circ}$ and $25^{\circ}, R_{v}$ and $R_{l}$ in between 0.1 to 0.4 .

The inversion process has been carried out initially by using FE results. The ultrasound was generated at 40 different nodes located from $45 \mathrm{~mm}$ to $107.4 \mathrm{~mm}$ to the right of the centre of the weld (see Figure 5), with pitch of $1.6 \mathrm{~mm}$, and in each generation the signal was monitored at 60 locations from $-50 \mathrm{~mm}$ to $-144.4 \mathrm{~mm}$ with the same pitch, to the left of the weld. Therefore the time of flight results were obtained in the format of a $40 \times 60$ matrix, which was then used to compare with an equivalent matrix of results from the ray-tracing model, using a fitness function as shown in equation 5 . In each ray-tracing computation, an angular sweep was performed at the position of the generator. Multiple rays were calculated for a range of shooting angles, delivering a series of arrivals along the surface where the monitoring points are located. The time of flight result at the positions of the monitors were then obtained by interpolation between the nearest arriving rays. Different sets of initialisation values were chosen in the inversion model, all leading to the convergence of the fitness function shown in equation 5. Figure 10 shows the inversion results from the genetic algorithm for 100 generations together with the best fitness value from the fitness equation. It was found that after 100 generations, the four MINA parameters converged to the following values: $\theta_{B}=17.08^{\circ}, \theta_{C}=0.82^{\circ}, R_{v}=0.165, R_{l}=0.311$, which agreed very well with the weld map used in the FE simulations $\left(\theta_{B}=17.5^{\circ}, \theta_{C}=0, R_{v}=\right.$ $\left.0.15, R_{l}=0.335\right)$. 


\section{Experiment}

\subsection{Experimental setup and signal processing}

Experiments were carried out to validate the weld map inversion process. The experimental setup is shown in Figure 11(a). A pair of 32 element ultrasonic phased array transducers (Imasonic A102 with $1.6 \mathrm{~mm}$ pitch) with $2 \mathrm{MHz}$ central frequency were placed across the weld. A M2M MultiX LF 64 channel array controller was used to generate and receive ultrasonic signals. In order to maximize the energy of shear waves in the generation, a $34.7^{\circ}$ Rexolite wedge was attached to the transmission array. The transmission array was placed as close to the weld cap region as possible and the receiving array was placed $60 \mathrm{~mm}$ away from the transmission array. The ultrasound was generated by a $100 \mathrm{~V}$ amplitude pulse at each of the channels from the transmission array. It passed through the weld, reflected from the back face of the sample and was received by all channels in the receiving array.

Figure 11(b) shows a typical B scan signal which was generated from element 1 of the transmission array and received from all elements in the receiving array. The $\mathrm{x}$ axis of the figure shows the receiver array element number (from left to right) according to the orientation seen in part (a) of the figure. The y axis shows the digitized sample number, corresponding to arrival time. The grey scale represents the signal amplitude. From the figure it can be seen that both longitudinal and shear modes were captured by the receiving array, which can be distinguished by the slope of their waveforms. The shear wave, which is stronger than the longitudinal wave due to the existence of the wedge, has proven to be more sensitive to the weld properties, therefore it needs to be extracted for the inversion process. However, since the shear wave is much slower than the longitudinal wave, it may arrive at the same time as other components, such as a longitudinal wave or mode con- 
verted wave arriving by a different path, which creates challenges to extract the accurate waveform of the shear wave.

As an example, Figure 12(a) shows an A scan signal (generated from the 10th element of the transmission array and received from the 5th element of the receiving array), where the shear wave is inside a multiple waveform at around $65 \mu \mathrm{s}$. To extract the shear wave, the "CLEAN" algorithm [27] was applied to the signal, which assumes that the multi-path signal is composed of a finite number of scaled, delayed and phased-shifted replicas of the transmitted signal. The algorithm tends to extract the dominant component of the signal, and has been applied successfully by other researchers $[8,28]$ to extract the longitudinal mode when this arrives early and strongly. Here, we use it to pick up the dominant shear mode. This is possible because we have used the wedge deliberately to enhance the amplitude of the shear mode in comparison with the longitudinal mode.

Figure 12(b) shows the resulting signal after this signal processing procedure.

\subsection{Inversion based on experimental results}

The inversion process has been performed to find the best fit parameters in the MINA model for the real weld, using the experimental measurements. The procedure is similar to that which was performed in the FE study, although there was a slight change in the forward model to take the wedge into account. The material properties of the austenitic weld used in the experiment were determined using the Electron BackScatter Diffraction (EBSD)based method, Orientation Distribution Functions (ODFs) were determined from the EBSD data and combined with the literature value for the single crystal stiffness tensor of monocrystal Fe-18\% Cr [29]. The method is described in more detail in [10]. The calculated values are shown in Table 1; 
these are slightly different from the values from the literature that we used earlier.

Figure 13 illustrates the process of the inversion using experimental data. Figure 13(a) shows ray paths from one of the excitation elements with different shooting angles. Shear waves are generated through the refraction from the wedge to the metal. The rays propagate through the weld, reflect at the back wall and terminate on the other side of the weld. Figure 13(b) shows the time of flight results with respect to the element locations in the receiving array from a single excitation position, which were obtained by interpolating the time of flight results from all the termination positions on the surface, in the same manner as was discussed earlier for the FE inversion. Figure 13(c) presents the time of flight results for all the combination of 32 generation elements and 32 receiving elements; the grey scale of each element in the figure represents the time of flight. These arrival times can be compared directly with those obtained from the experiment shown in Figure 13(d) for the inversion process. Figure 13(c) also indicates that in some regions the time of flight results are not available, because the rays terminate at the local boundaries inside the weld [3]. The inversion procedure is the same as in previous examples, which is to find the best match in time of flight between the forward models and experiments based on global optimization using the genetic algorithm.

After 100 iterations, the four MINA parameters have converged to the following values: $\theta_{B}=20.5^{\circ}, \theta_{C}=5.63^{\circ}, R_{v}=0.173, R_{l}=0.362$. Figure 14 shows the the corresponding macrograph from the optimized MINA parameters and the comparison to the measured map from the macrograph. It suggests that the difference in the grain orientation map is less than 20 degrees for most of the regions inside the weld. Given that the intention is to provide a robust inversion that is correct in general, even if imprecise in local 
detail, this is considered to be an acceptable agreement. The significance of this will need to wait for the assessment of the effectiveness of correcting array images in practical applications, but it is already reasonable to expect a large improvement over the assumptions that currently need to be made in many inspections that the weld material is isotropic and homogeneous.

\section{Conclusions}

Variations in stiffness local to welds can significantly affect the passage of ultrasound waves and thereby, if unaccounted for, interfere with non destructive evaluation of near-weld regions. Since these are likely locations for defects this represents a significant challenge for non destructive inspection. In this paper we have shown that we can use ultrasonic arrays to reconstruct an estimated weld map for an example weld that is representative of power plant components, defining the orientation of the stiffness tensor map in a smoothly varying form over the weld region. Furthermore we have done this using one of the validated functions in the literature that involves only a few key parameters that can be related to the welding procedure.

Ray-tracing techniques have been applied in forward models to calculate the ultrasound propagation through the weld. Finite Element simulations have been carried out to validate the ray model and to investigate a good approach for the inversion process. It has been demonstrated using the FE studies that the shear mode polarised in the plane of the cross section (SV mode), which is more sensitive than the longitudinal mode to variations of the weld properties, can be detected satisfactorily provided the monitor locations are well chosen, and then used to drive the inversion. Inverse models, based on global optimization, have been carried out to compose the estimated weld map for both FE simulations and experiments, showing good agreement.

The inversion results from the real sample, although reasonable, are not 
as good as those from the FE simulations, and this is because a number of approximations were made in the modeling of the weld properties. The material properties were considered to be transversely isotropic, and symmetrically orientated with respect to the plane of the cross-section, so that a two dimensional model can be applied, however in reality the orientation of the polycrystal grains in the weld may be tilted slightly out of the plane [10]. A more accurate approach would be to consider the material properties of the weld to be orthotropic [30], and apply a three dimensional model to describe the weld map. Another assumption in the weld modelling is to consider the elastic constants to be the same everywhere in the weld, with the only variable being the rotation angle of the stiffness matrix according to the positions. This is an oversimplification, in reality it is possible that the elastic constants could vary slightly in the weld region. Therefore it would be more accurate if this could be taken into account in the mapping process, i.e. in each position of the weld the variable would be the elastic constants plus a rotation angle. These ideas could be investigated for better accuracy in the inversion of experimental measurements. However, such modifications would add considerably to the complexity of the process, and, given that the model presented in this work has already demonstrated reasonably good performance, it is questionable whether the increase in complexity and possible reduction in robustness would be worthwhile.

\section{Acknowledgements}

The authors are grateful to UK Research Centre in Non-Destructive Evaluation (RCNDE), the Engineering and Physical Science Research Council (EPSRC grant numbers EP/G042772/1 and EP/G045720/1), Rolls-Royce Nuclear, E.ON and Petrobras for funding this work. 


\section{References}

[1] J. A. Ogilvy, Computerized ultrasonic ray tracing in austenitic steel, NDT Int. 18 (2) (1985) 67-77.

[2] V. Schmitz, F. Walte, S. V. Chakhlov, 3D ray tracing in austenite materials, NDT \& E International 32 (4) (1999) 201-213.

[3] G. D. Connolly, M. J. S. Lowe, J. A. G. Temple, S. I. Rokhlin, Correction of ultrasonic array images to improve reflector sizing and location in inhomogeneous materials using a ray-tracing model, J. Acoust. Soc. Am. 125 (5) (2010) 2802-2812.

[4] K. Jezzine, A. Gardahaut, N. Leymarie, S. Chatillon, Evaluation of ray-based methods for the simulation of welds inspection, in: D. O. Thompson, D. E. Chimenti (Eds.), Review of Progress in Quantitative NDE, Vol. 39, American Institute of Physics, New York, 2013, pp. 10731080.

[5] B. Chassignole, D. Villard, M. Dubuget, J. C. Baboux, R. E. Guerjouma, Characterization of austenitic stainless steel welds for ultrasonic NDT, in: D. O. Thompson, D. E. Chimenti (Eds.), Review of Progress in Quantitative NDE, Vol. 19, American Institute of Physics, New York, 2000, pp. 1325-1332.

[6] J. Moysan, A. Apfel, G. Corneloup, B. Chassignole, Modelling the grain orientation of austenitic stainless steel multipass welds to improve ultrasonic assessment of structural integrity, Int. J. Pres. Ves. \& Piping 80 (2003) 77-85.

[7] C. Gueudre, L. Le Marrec, J. Moysan, B. Chassignole, Direct model optimisation for data inversion. application to ultrasonic characterisation of heterogeneous welds, NDT \& E International 42 (2009) 47-55. 
[8] J. Zhang, A. Hunter, B. W. Drinkwater, P. D. Wilcox, Monte Carlo inversion of ultrasonic array data to map anisotropic weld properties, IEEE Trans. Ultrason. Ferroelect. Freq. Contr. 59 (11) (2012) 24872497.

[9] O. Nowers, D. J. Duxbury, J. Zhang, B. W. Drinkwater, Novel raytracing algorithms in nde: Application of dijkstra and $\mathrm{A}^{*}$ algorithms to the inspection of an anisotropic weld, NDT \& E International 61 (2014) $58-66$.

[10] A. F. Mark, Z. Fan, F. Azough, M. J. S. Lowe, P. J. Withers, Investigation of the elastic/crystallographic anisotropy of welds for improved ultrasonic inspections, Materials Characterization 98 (2014) 47-53.

[11] A. Apfel, J. Moysan, G. Corneloup, B. Chassignole, Simulation of the influence of the grain orientation on ultrasounds, in: Proceedings of 16th WCNDT, Montreal, 2004.

[12] J. A. Ogilvy, The ultrasonic reflection properties of planar defects within austenitic welds, Ultrasonics 26 (6) (1986) 318-327.

[13] K. J. Langenberg, R. Hannemann, T. Kaczorowski, R. Marklein, B. Koehler, C. Schurig, F. Walte, Application of modeling techniques for ultrasonic austenitic weld inspection, NDT \& E 33 (2000) 465-480.

[14] M. Spies, Modeling of transducer fields in inhomogeneous anisotropic materials using Gaussian beam superposition, NDT \& E International 33 (2000) 155-162.

[15] M. G. Silk, Computer model for ultrasonic propagation in complex orthotropic structures, Ultrasonics 19 (1981) 208-212. 
[16] R. S. Long, J. Russel, P. Cawley, Throughweld ultrasonic phased array inspection using full matrix capture, in: D. O. Thompson, D. E. Chimenti (Eds.), Review of Progress in Quantitative NDE, Vol. 29, American Institute of Physics, New York, 2010, pp. 918-925.

[17] J. Moysan, G. Corneloup, B. Chassignole, C. Gueudr, M. Ploix, Modelling welded material for ultrasonic testing using mina: theory and applications, in: D. O. Thompson, D. E. Chimenti (Eds.), Review of Progress in Quantitative NDE, Vol. 38, American Institute of Physics, New York, 2012, pp. 1219-1226.

[18] C. Gueudre, L. Le Marrec, M. Chekroun, J. Moysan, B. Chassignole, G. Corneloup, Determination of the order of passes of an austenitic weld by optimization of an inversion process of ultrasound data, in: D. O. Thompson, D. E. Chimenti (Eds.), Review of Progress in Quantitative NDE, Vol. 1335, American Institute of Physics, New York, 2011, pp. 639-646.

[19] B. A. Cervenŷ, Seismic Ray Theory, Cambridge University Press, Cambridge UK, 2001.

[20] S. I. Rokhlin, K. Bolland, L. Adler, Reflection and refraction of elastic waves on a plane interface between two generally anisotropic media, J. Acoust. Soc. Am. 79 (1986) 908-918.

[21] J. A. G. Temple, Modelling the propagation and scattering of elastic waves in inhogeneous anisotropic media, J. Phys. D Appl. Phys. 21 (1988) 859-874.

[22] ABAQUS, Analysis User's Manual, Version 6.9, http://www.simulia.com/, 2009. 
[23] R. Rajagopal, M. Drozdz, E. A. Skelton, M. J. S. Lowe, R. V. Craster, On the use of absorbing layers to simulate the propagation of elastic waves in unbounded media using commercially available finite element packages, NDT \& E International 51 (2012) 30-40.

[24] E. Bécache, P. Joly, C. Tsogka, An analysis of new mixed finite elements for the approximation of wave propagation problems, SIAM J. Numer. Anal. 37 (2000) 1053-1084.

[25] J. H. Holland, Adaptation in natural and artificial systems, Michigan Press, 1975.

[26] D. L. Carroll, FORTRAN genetic algorithm driver, Version 1.7, http://iri.columbia.edu/ines/PeterDroogers/Genetic_Algorithm/, last accessed July 2013.

[27] P. Gough, A fast spectral estimation algorithm based on the FFT, IEEE Trans. Signal Process. 42 (6) (1994) 1317-1322.

[28] A. J. Hunter, B. W. Drinkwater, J. Zhang, P. D. Wilcox, A study into the effects of an austenitic weld on ultrasonic array imaging performance, in: D. O. Thompson, D. E. Chimenti (Eds.), Review of Progress in Quantitative NDE, Vol. 1335, American Institute of Physics, New York, 2011, pp. 1063-1070.

[29] H. Ogi, H. Niho, M. Hirao, Elastic-stiffness distribution on dual-phase stainless steel studied by resonance ultrasound microscopy, Acta Materialia 54 (2006) 4143-4148.

[30] B. Chassignole, R. E. Guerjouma, M.-A. Ploix, T. Fouque, Ultrasonic and structural characterization of anisotropic austenitic stainless steel welds: Towards a higher reliability in ultrasonic non-destructive testing, NDT \& E International 43 (4) (2010) 273-282. 


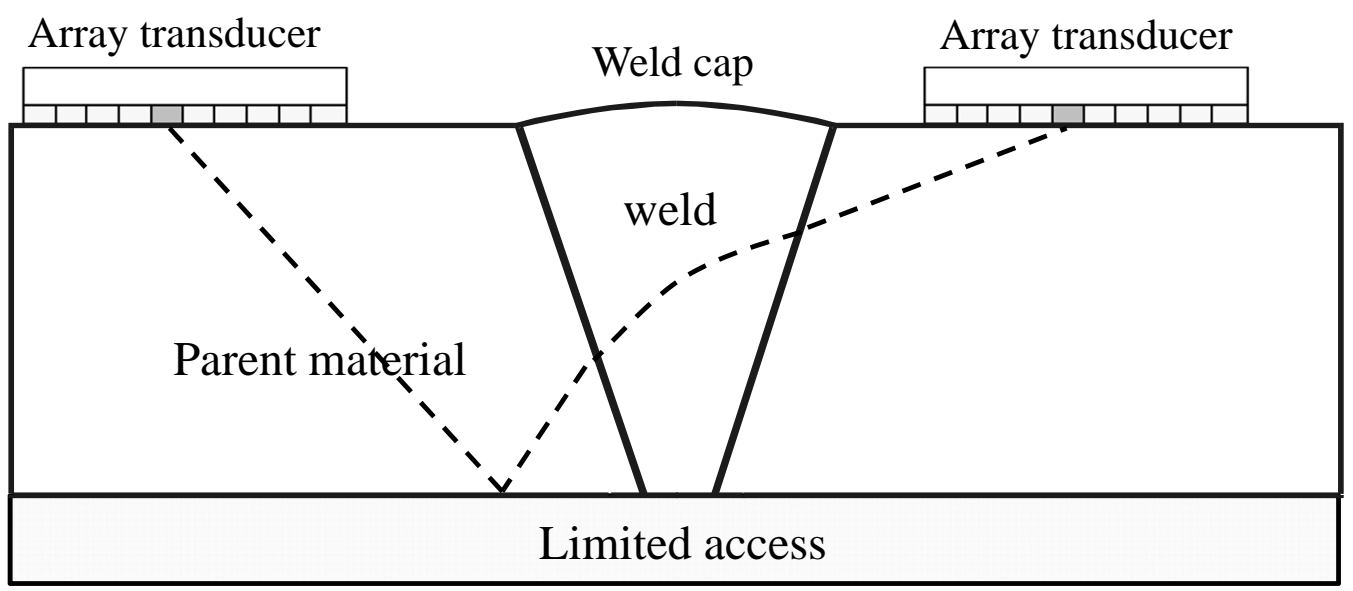

Figure 1: Schematic showing the characterisation of a weld using ultrasonic arrays. The dashed line illustrates the path of one of the multiple rays from the array elements of the transmitter to those of the receiver. 

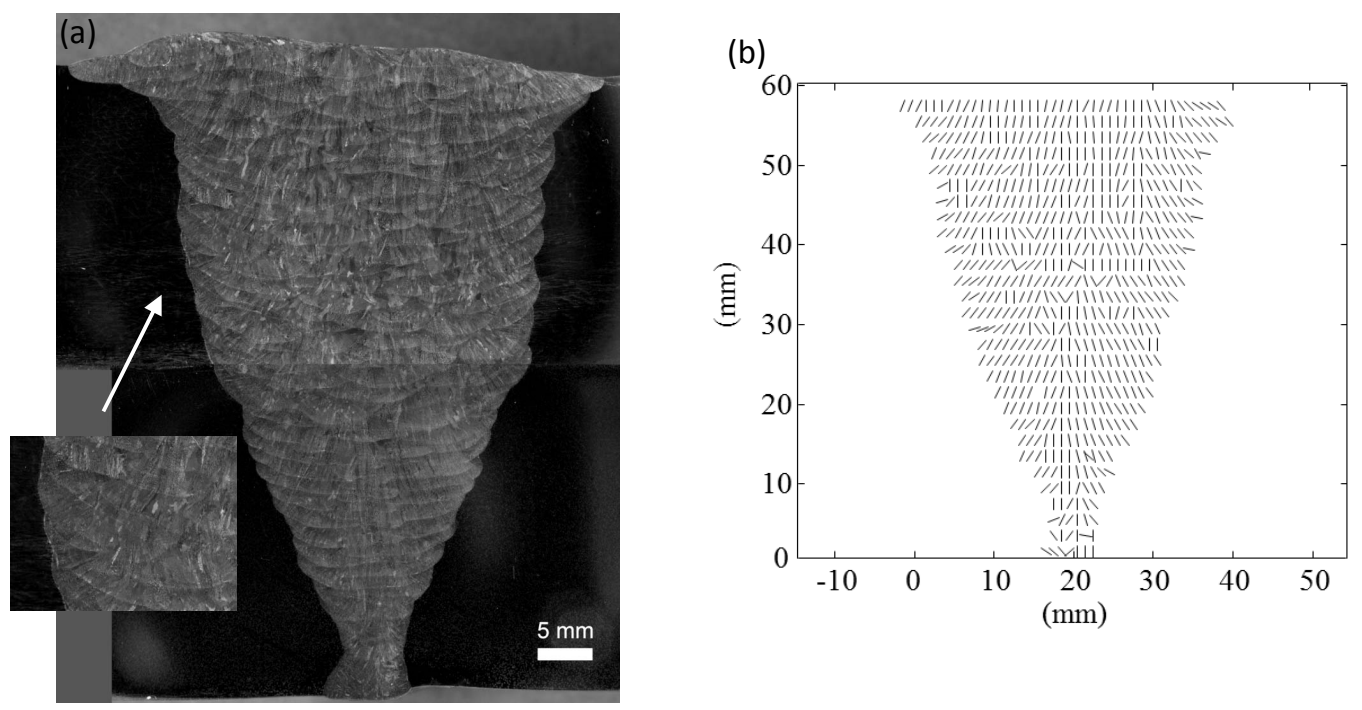

Figure 2: (a) Macrograph of a weld sample used for the study with inset to show an example of the grain structure; this is a joint between $60 \mathrm{~mm}$-thick plates of 304L austenitic stainless steel, with 308 stainless steel composing weld; (b) grain orientation map of the weld inferred from the directions seen in the macrograph using a $1 \times 2 \mathrm{~mm}^{2}$ mesh. 


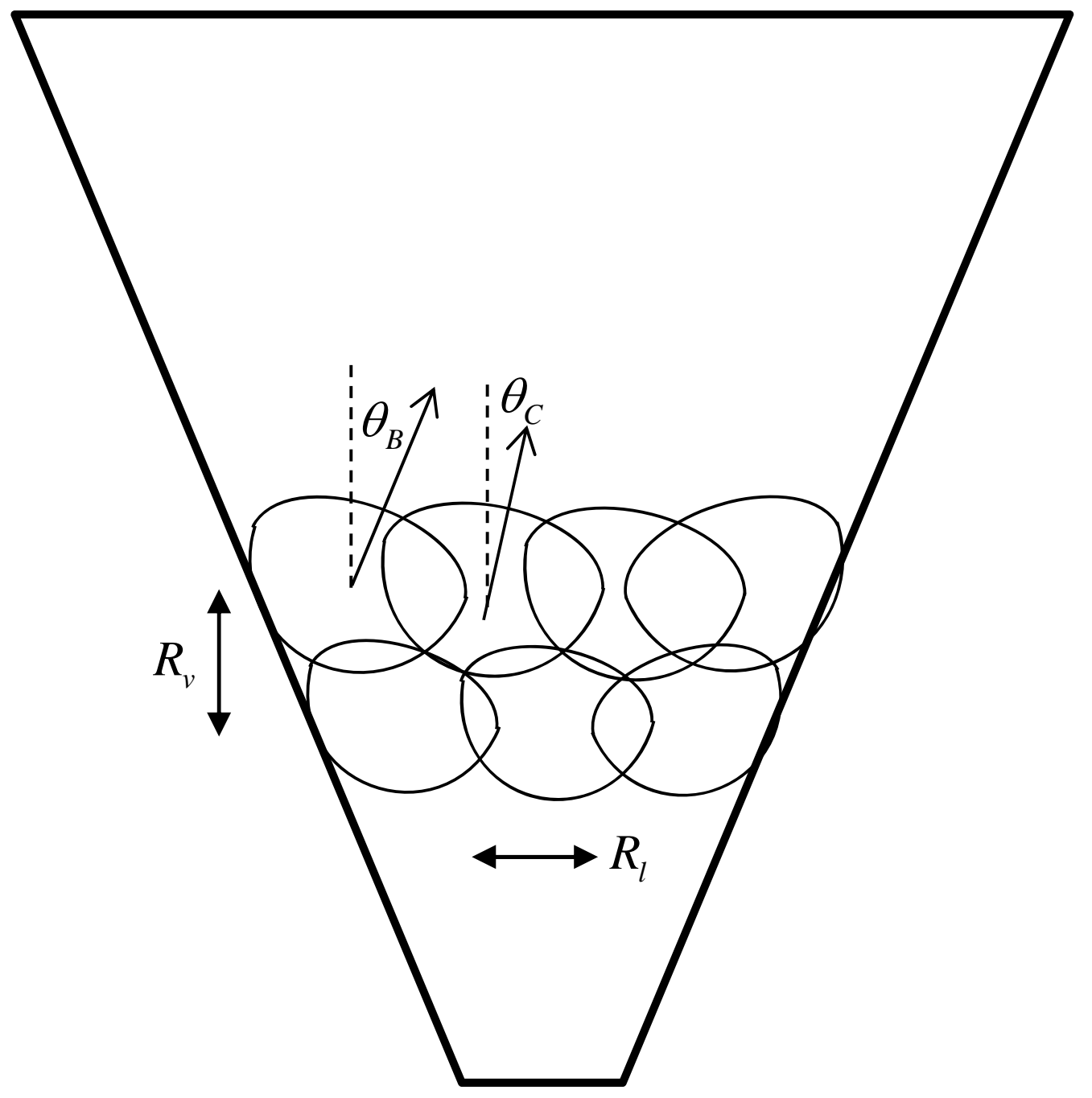

Figure 3: Schematic of MINA [6] modelling, showing the key parameters that were implemented as the unknowns in the inversion model. 

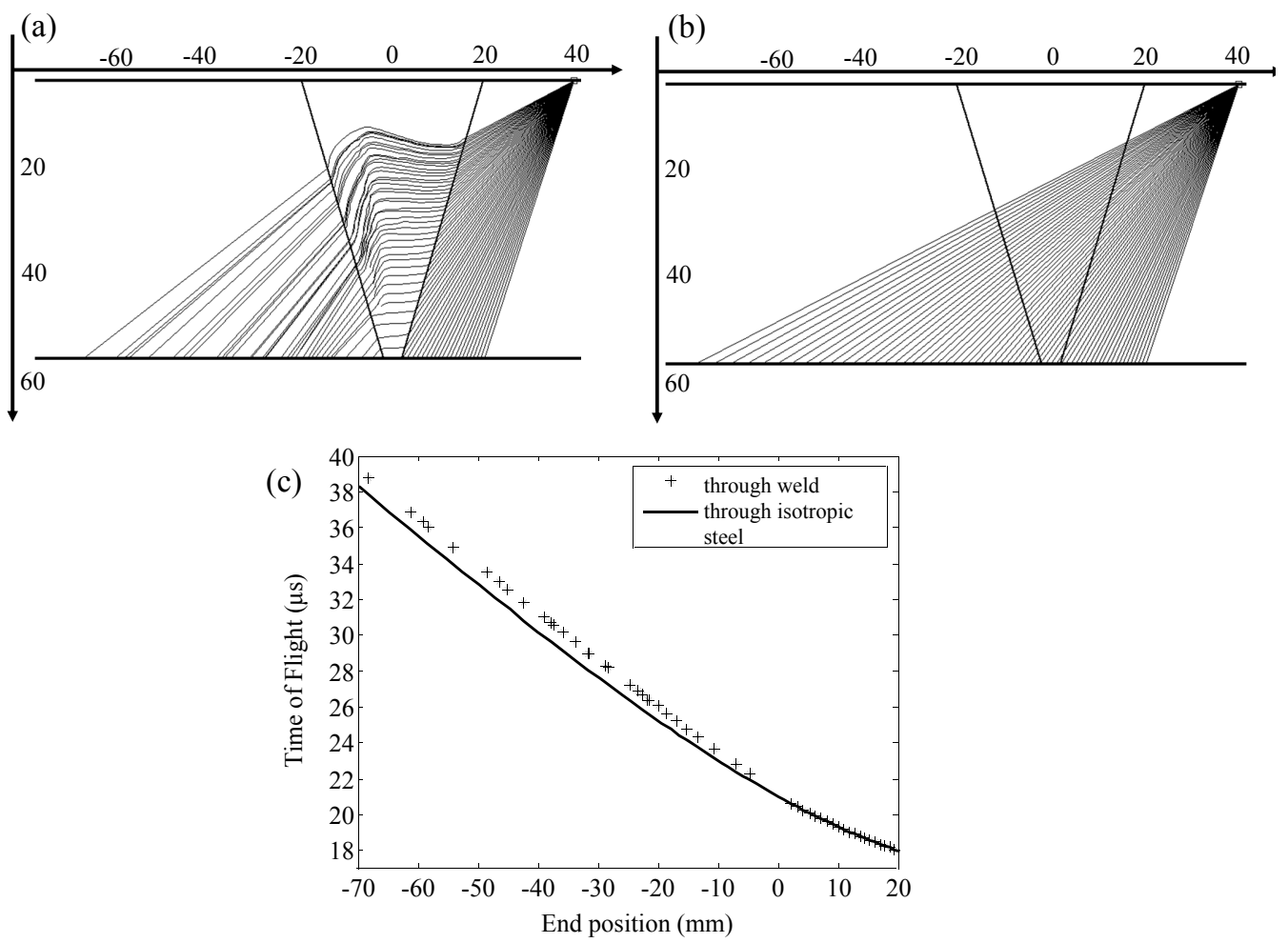

Figure 4: Ray model simulations: (a) ray paths of shear waves through the weld; (b) ray paths of shear waves if the weld is assumed to be isotopic; (c) time of flight of the rays from the source location to the end position at the bottom of the model, showing comparison of the travel times for the deviated and straight ray solutions. 


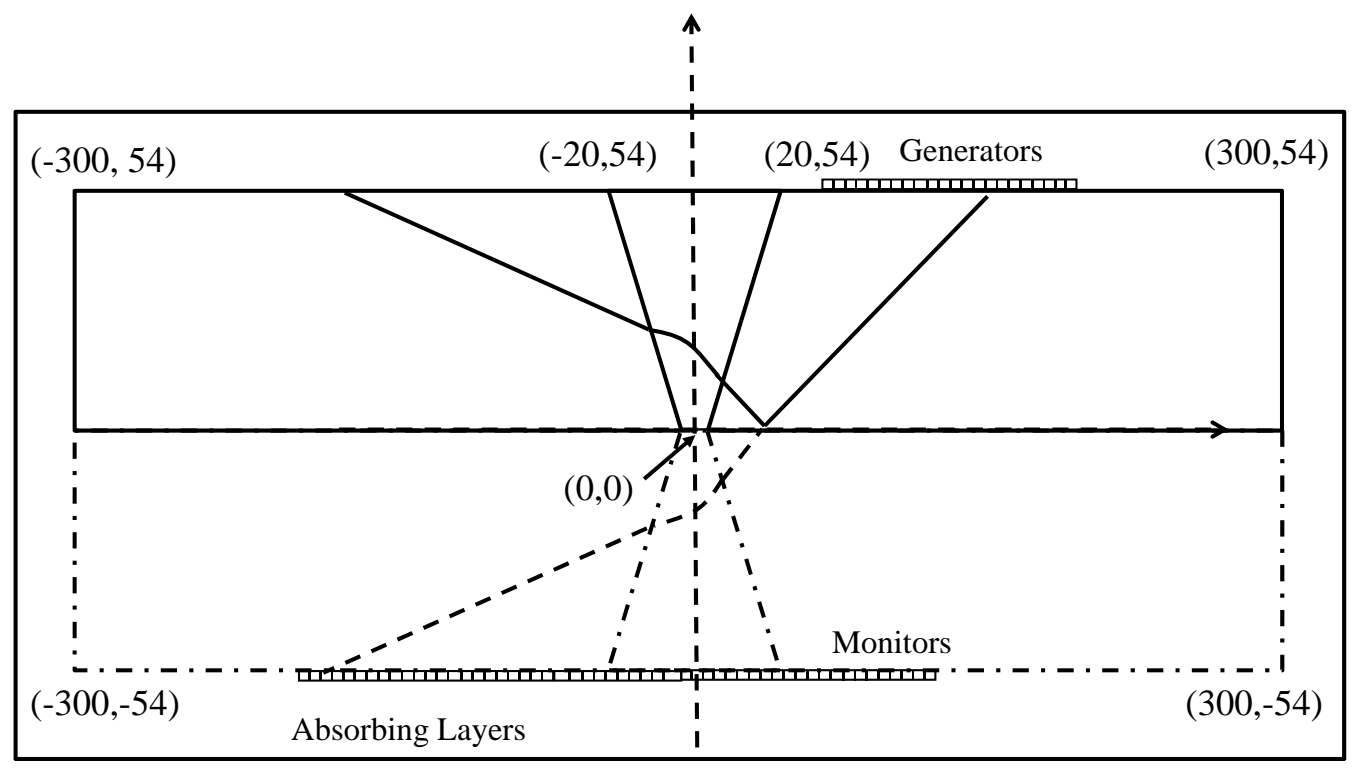

Figure 5: Schematic of the FE model (not to scale). The dashed lines in the lower half of the model represent the mirror image of the weld, included in the model as a convenience so that the simulation results would not include mode converted waves from the lower surface of the structure. 

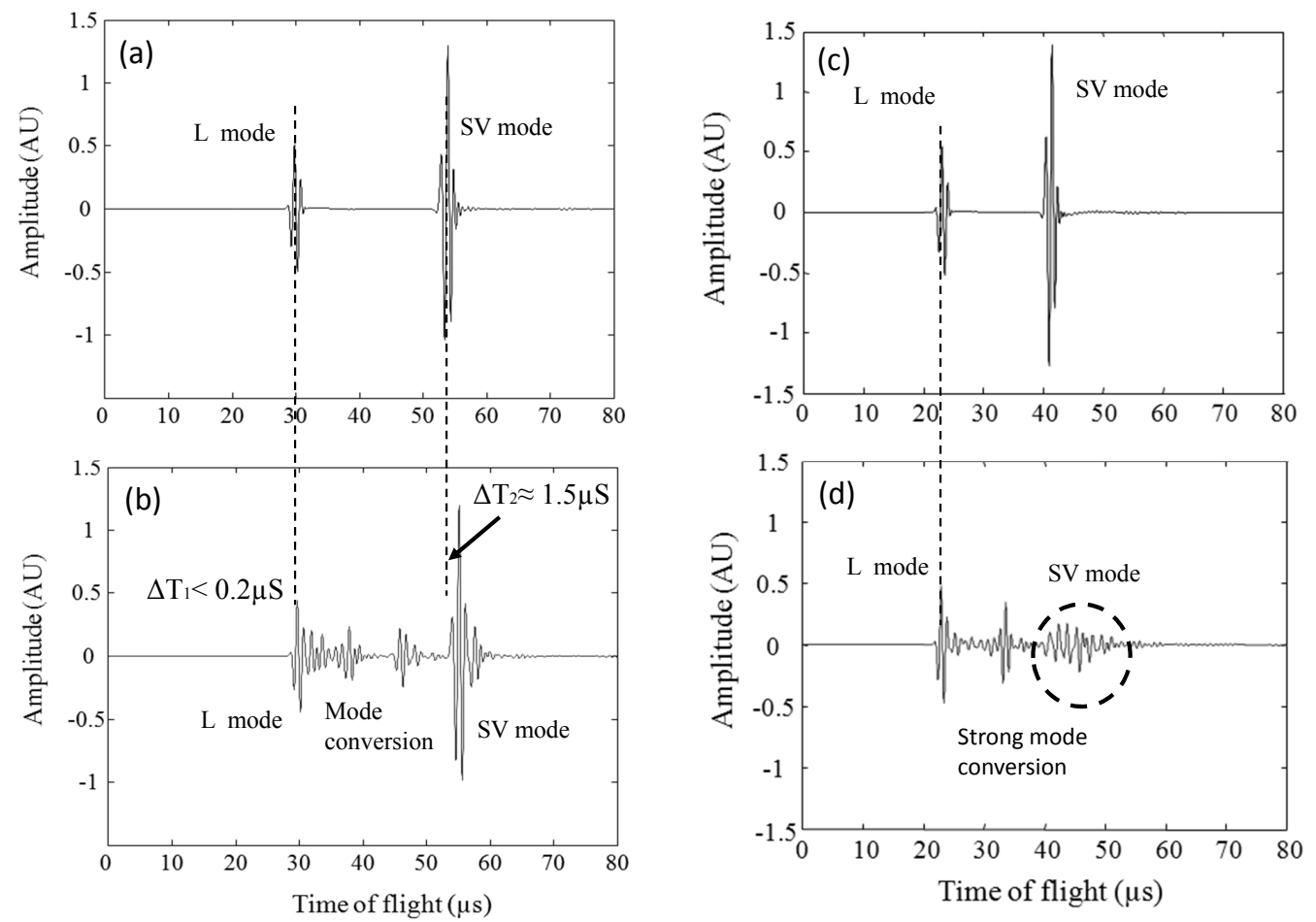

Figure 6: FE simulated signals of ultrasound received at $100 \mathrm{~mm}$ through parent material (a) and through weld (b); simulated signals of ultrasound received at $22 \mathrm{~mm}$ through parent material (c) and through weld (d). 
(a)
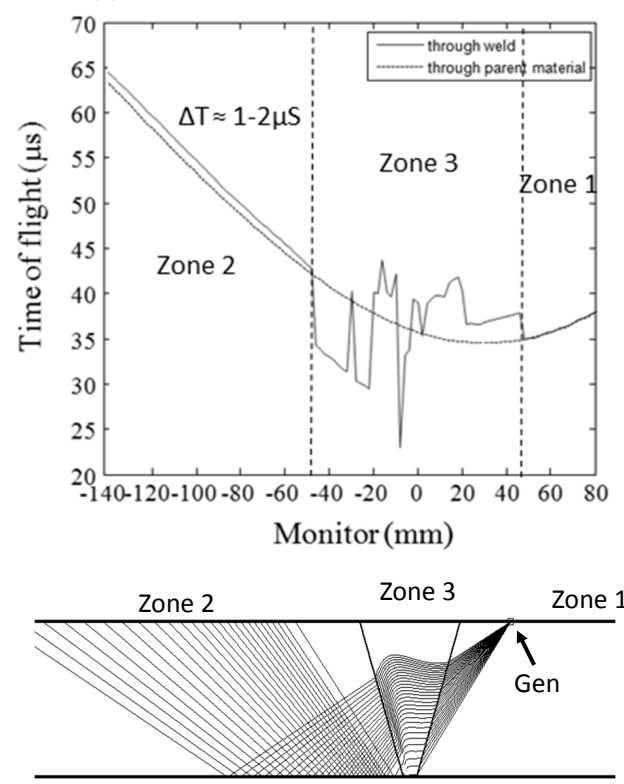

(b)
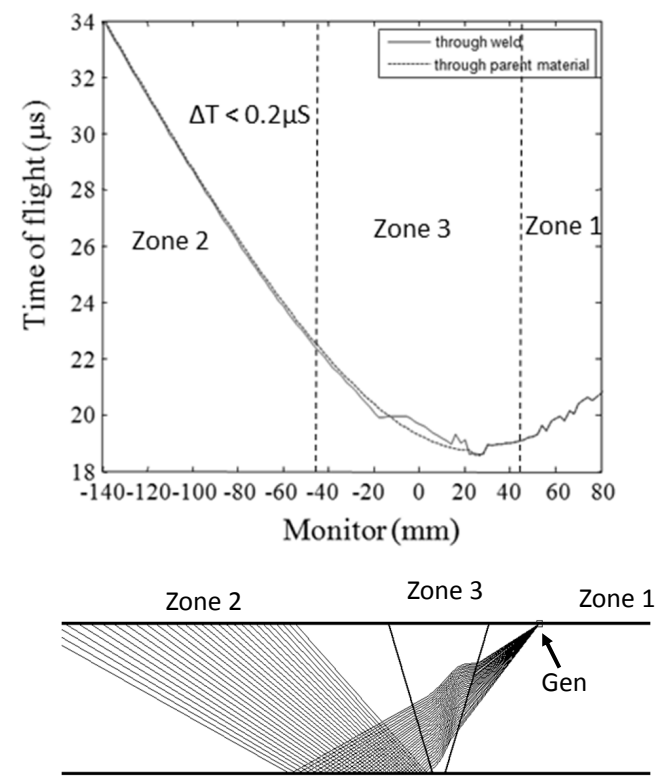

Figure 7: Time of flight results with respect to monitor positions of shear (a) and longitudinal (b) modes, with solid lines representing results from ultrasound propagation through the weld and dotted lines representing that through isotropic steel. 


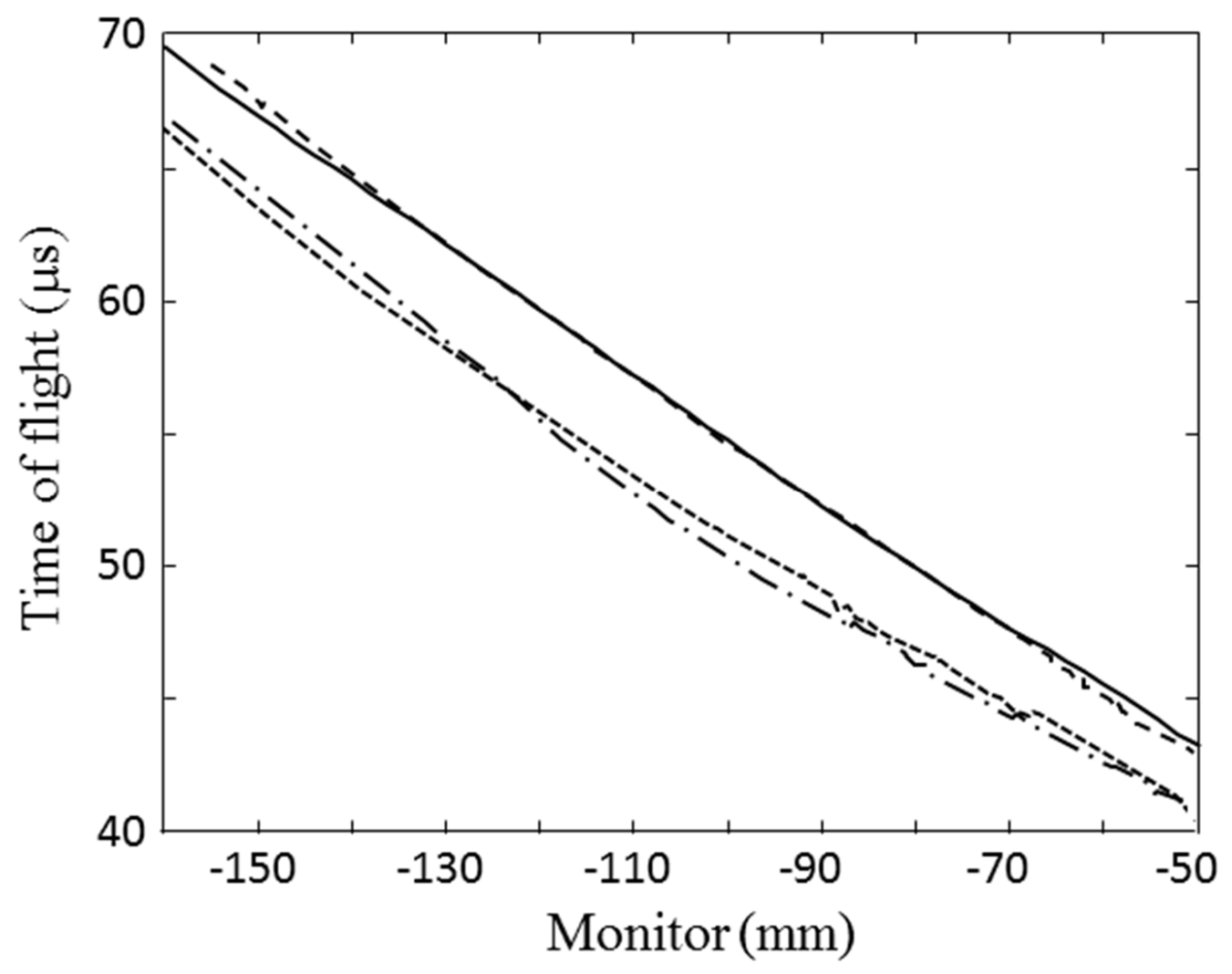

Figure 8: Comparison of finite element results of different weld maps with ray-tracing results. The solid line is the result from FE simulation, and the dashed line represents results from ray-tracing with the weld map generated with the same MINA parameters $\left(\theta_{B}=17.5^{\circ}, \theta_{C}=0, R_{v}=0.15, R_{l}=\right.$ 0.335). The dotted and dash-dot lines are the raytracing results with weld maps generated with the following alternative MINA parameters respectively: $\theta_{B}=40^{\circ}, \theta_{C}=30^{\circ}, R_{v}=0.5, R_{l}=0.2$ and $\theta_{B}=30^{\circ}, \theta_{C}=20^{\circ}, R_{v}=$ $0.3, R_{l}=0.2$. 


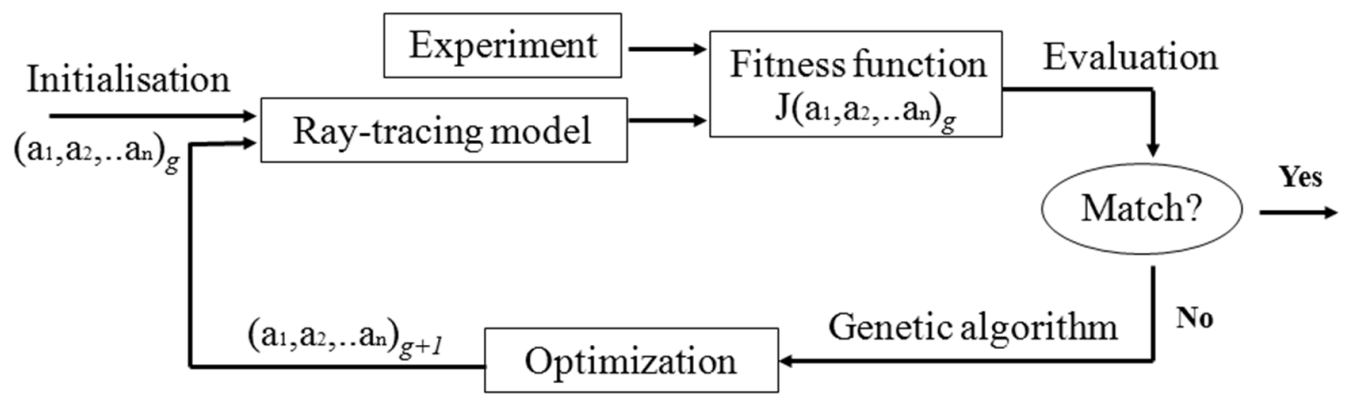

Figure 9: Inversion model using genetic algorithm. 

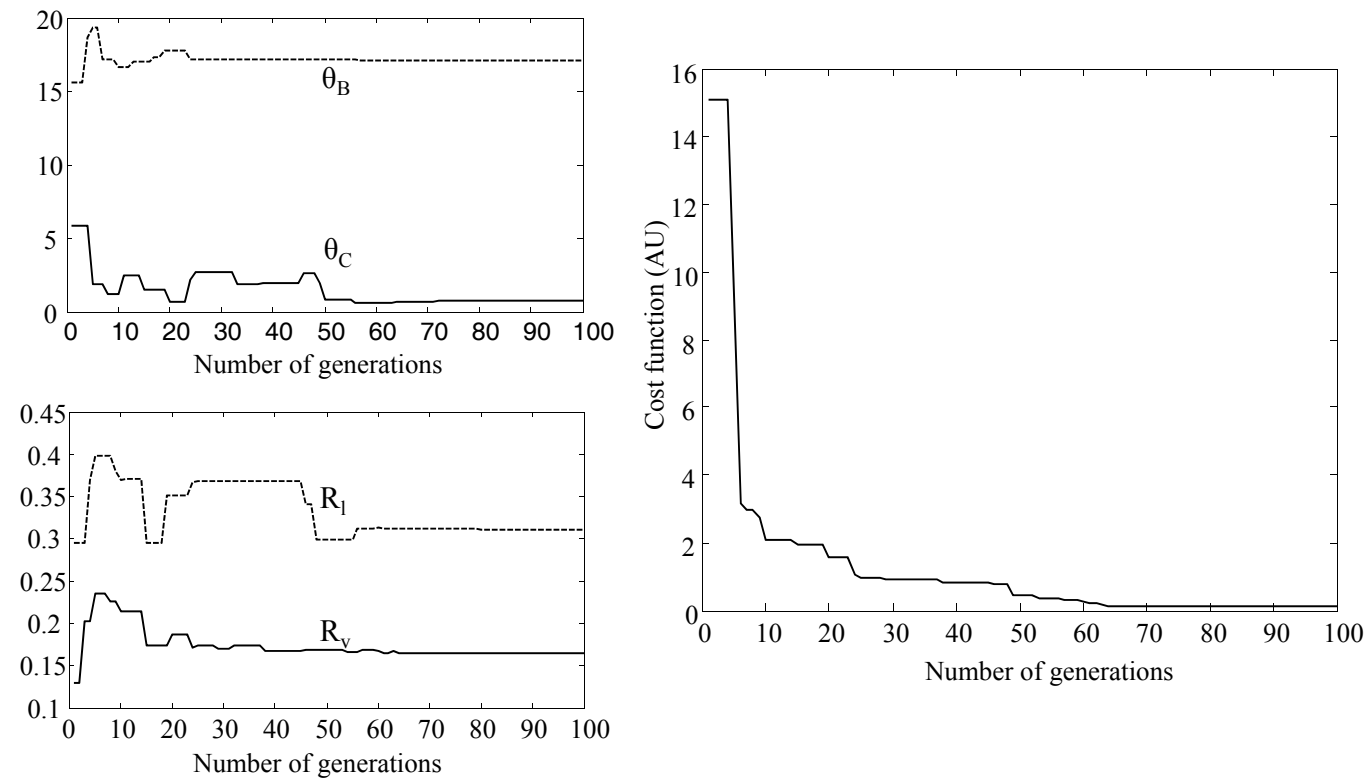

Figure 10: Inversion results (left) of the four MINA parameters, and cost function (right), showing convergence over 100 generations. 
(a)

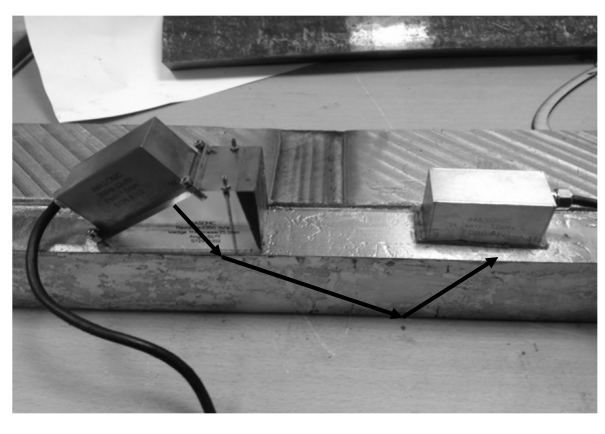

(b)

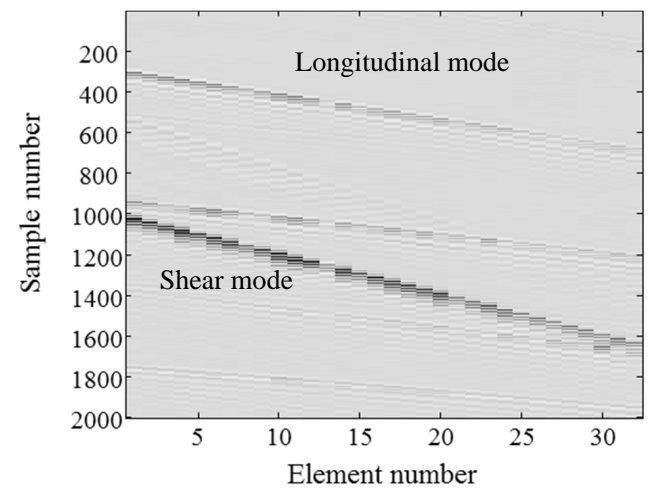

Figure 11: a) Experimental setup for weld map characterization; (b) typical B scan signal map from one generating position. 
(a)

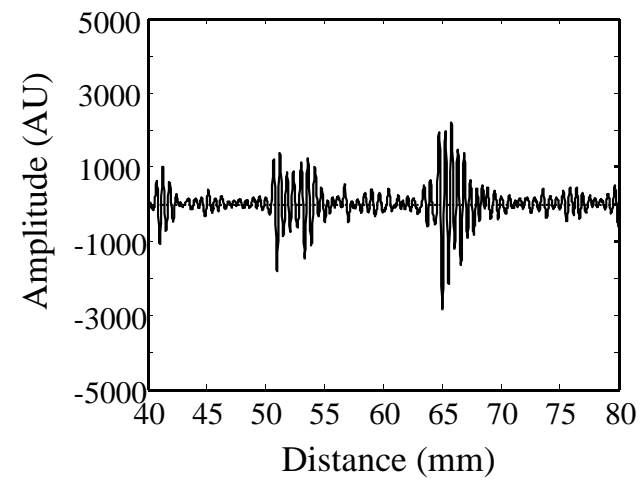

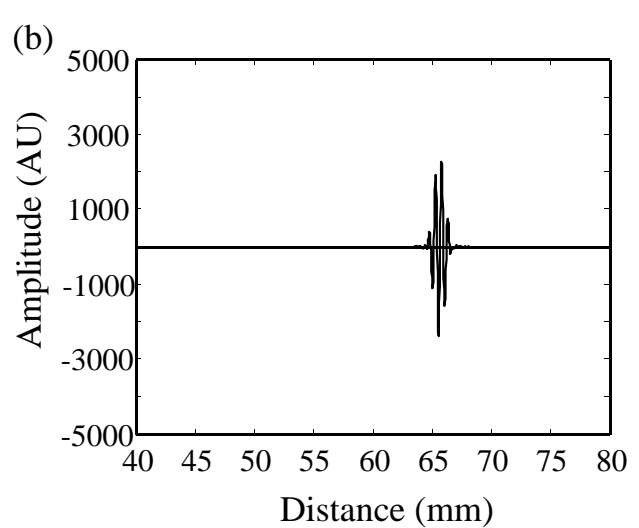

Figure 12: a) A scan signal received from one element; (b) extracted shear wave using CLEAN algorithm. 


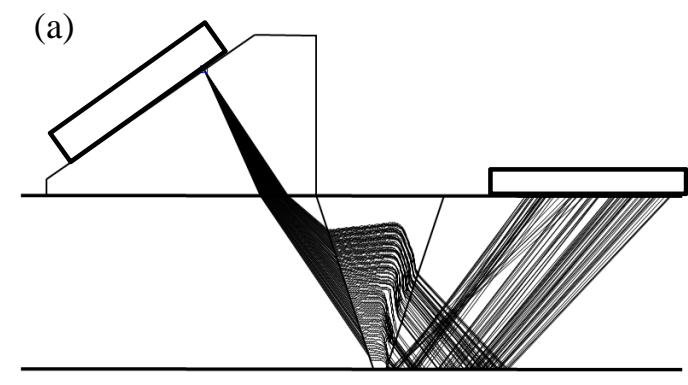

(b)

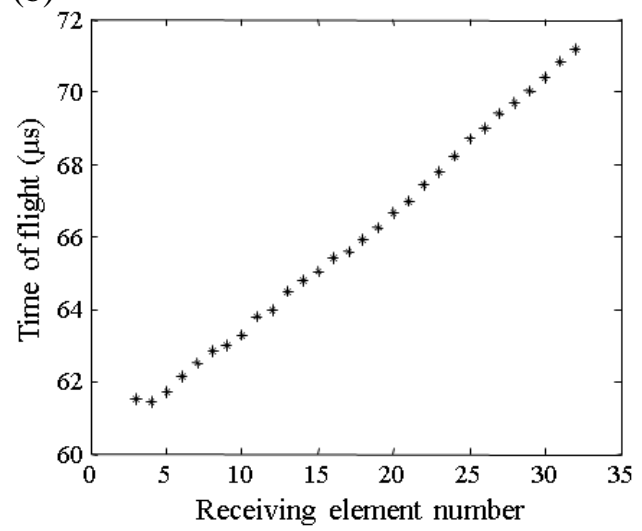

(c) Time of flight (simulation)

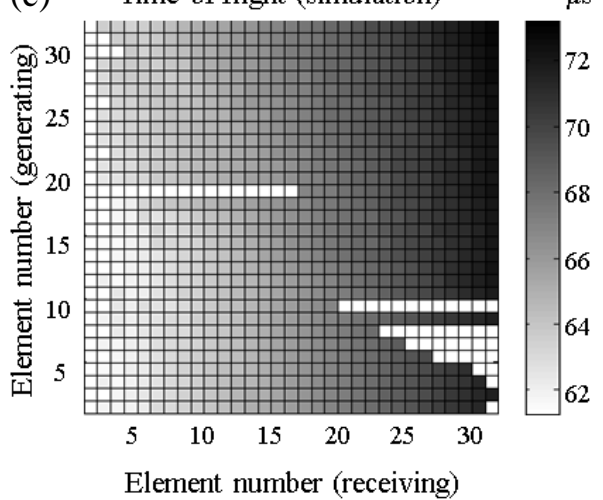

(d) Time of flight (experiment)

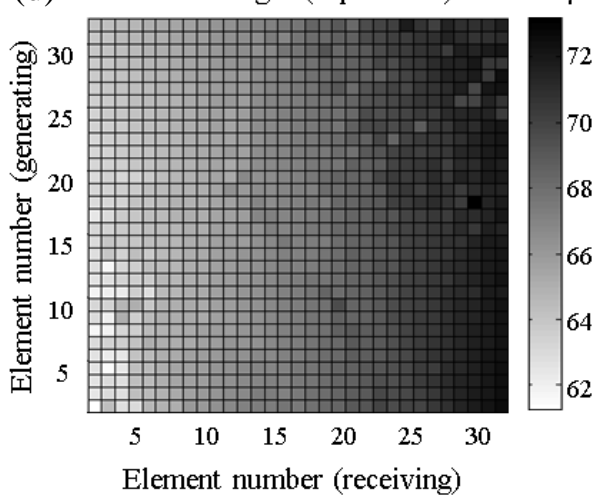

Figure 13: (a) Ray-tracing forward model for one excitation element with different shooting angles ; (b) time of flight results with respect to the element locations in the receiving array from a single excitation position; (c) time of flight (grey scale) results for all elements by ray-tracing simulations (d) time of flight results for all elements obtained in experimental measurements. 

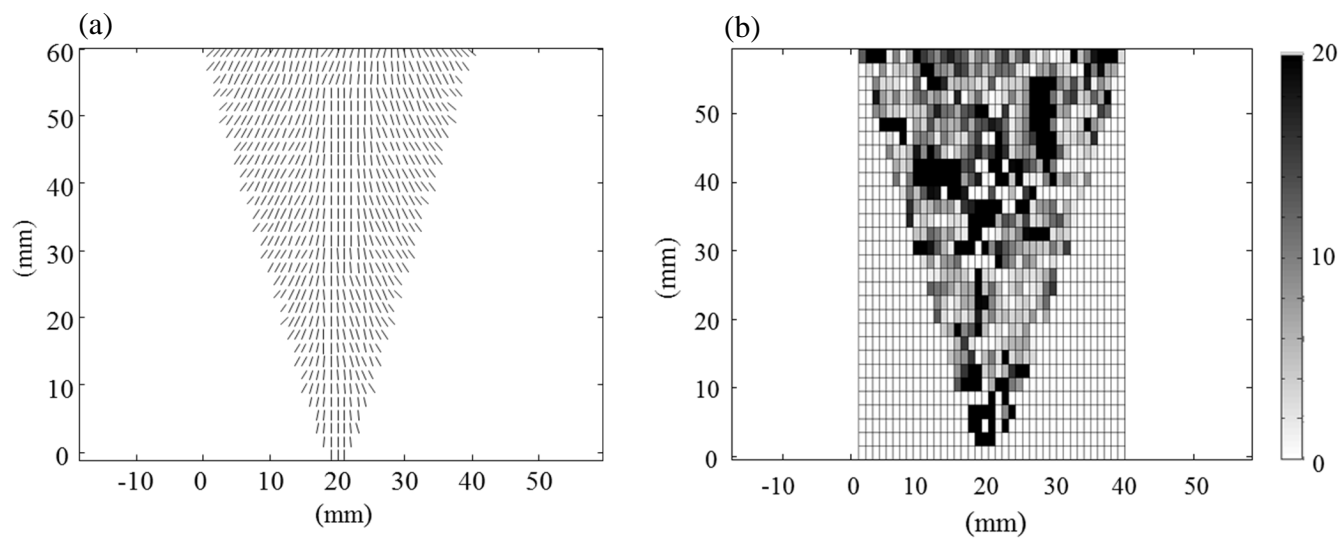

Figure 14: (a) Weld map for the real weld based on optimized MINA parameters obtained from the inversion of the experimental measurements; (b) comparison of the weld map obtained from inversion and that obtained directly from measurements from the macrograph (shown in figure 2(b)); the grey scale denotes angular error in degrees. 
Table 1: Material properties for isotropic and transversely isotropic steel, Voigt notation applies.

\begin{tabular}{|l|l|l|}
\hline $\begin{array}{l}\text { Isotropic steel } \\
\text { (parent plates) }\end{array}$ & $\begin{array}{l}\text { Anisotropic steel } \\
\text { (in weld models) }[21]\end{array}$ & $\begin{array}{l}\text { Anisotropic steel } \\
\text { (in experiment) }\end{array}$ \\
\hline $\mathrm{C} 11=283 \mathrm{GPa}$ & $\mathrm{C} 11=263 \mathrm{GPa}$ & $\mathrm{C} 11=245 \mathrm{GPa}$ \\
$\mathrm{C} 12=121 \mathrm{GPa}$ & $\mathrm{C} 12=98 \mathrm{GPa}$ & $\mathrm{C} 12=104 \mathrm{GPa}$ \\
$\mathrm{C} 13=121 \mathrm{GPa}$ & $\mathrm{C} 13=145 \mathrm{GPa}$ & $\mathrm{C} 13=121 \mathrm{GPa}$ \\
$\mathrm{C} 33=283 \mathrm{GPa}$ & $\mathrm{C} 33=216 \mathrm{GPa}$ & $\mathrm{C} 33=227 \mathrm{GPa}$ \\
$\mathrm{C} 44=80.7 \mathrm{GPa}$ & $\mathrm{C} 44=129 \mathrm{GPa}$ & $\mathrm{C} 44=105 \mathrm{GPa}$ \\
$\mathrm{C} 66=80.7 \mathrm{GPa}$ & $\mathrm{C} 66=82.5 \mathrm{GPa}$ & $\mathrm{C} 66=74.5 \mathrm{GPa}$ \\
Density $=7900 \mathrm{~kg} / \mathrm{m}^{3}$ & Density $=8010 \mathrm{~kg} / \mathrm{m}^{3}$ & Density $=8010 \mathrm{~kg} / \mathrm{m}^{3}$ \\
\hline
\end{tabular}

